\title{
Factors influencing the high rejection rates of HIV $1 / 2$ serology samples at Charlotte Maxeke Johannesburg Academic Hospital and the cost implications
}

\begin{tabular}{|c|c|}
\hline \multicolumn{2}{|c|}{$\begin{array}{l}\text { Authors: } \\
\text { Bhaveshan Reddy } y^{1,2} \text { (D) } \\
\text { Naseem Cassim }{ }^{2,3} \text { (I) } \\
\text { Florette Treurnicht } \\
\text { Zinhle Makatini }{ }^{1,2} \text { (D) }\end{array}$} \\
\hline \multicolumn{2}{|c|}{$\begin{array}{l}\text { Affiliations: } \\
{ }^{1} \text { Department of Virology, } \\
\text { Faculty of Health Science, } \\
\text { University of the } \\
\text { Witwatersrand, } \\
\text { Johannesburg, South Africa }\end{array}$} \\
\hline \multicolumn{2}{|c|}{$\begin{array}{l}{ }^{2} \text { National Health Laboratory } \\
\text { Service, Johannesburg, } \\
\text { South Africa }\end{array}$} \\
\hline \multicolumn{2}{|c|}{$\begin{array}{l}{ }^{3} \text { Department of Haematology } \\
\text { and Molecular Medicine, } \\
\text { Faculty of Health Sciences, } \\
\text { University of the } \\
\text { Witwatersrand, } \\
\text { Johannesburg, South Africa }\end{array}$} \\
\hline \multicolumn{2}{|c|}{$\begin{array}{l}\text { Corresponding author: } \\
\text { Bhaveshan Reddy, } \\
\text { reddybhaveshan@gmail.com }\end{array}$} \\
\hline \multicolumn{2}{|c|}{$\begin{array}{l}\text { Dates: } \\
\text { Received: } 05 \text { Oct. } 2021 \\
\text { Accepted: } 12 \text { Nov. } 2021 \\
\text { Published: } 11 \text { Jan. } 2022\end{array}$} \\
\hline \multicolumn{2}{|c|}{$\begin{array}{l}\text { How to cite this article: } \\
\text { Reddy B, Cassim N, Treurn } \\
\text { F, Makatini Z. Factors } \\
\text { influencing the high reject } \\
\text { rates of HIV } 1 / 2 \text { serology } \\
\text { samples at Charlotte Max } \\
\text { Johannesburg Academic } \\
\text { Hospital and the cost } \\
\text { implications. S Afr J HIV } \\
\text { Med. 2022;23(1), a1326. } \\
\text { https://doi.org/10.4102/ } \\
\text { sajhivmed.v23i1.1326 }\end{array}$} \\
\hline \multicolumn{2}{|l|}{ Read online: } \\
\hline 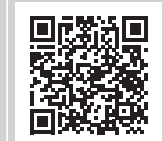 & $\begin{array}{l}\text { Scan this QR } \\
\text { code with your } \\
\text { smart phone or } \\
\text { mobile device } \\
\text { to read online. }\end{array}$ \\
\hline
\end{tabular}

\begin{abstract}
Background: HIV enzyme-linked immunosorbent assay (ELISA) is one of the most requested test sets within Virology and forms an essential part of patient management. Assessment of the rejection criteria is a key quality indicator, crucial for improving laboratory services and efficiency to ensure accurate and reliable results.
\end{abstract}

Objectives: The aim of this study was to identify the factors that influence the HIV $1 / 2$ serology rejection rates (RR) at Charlotte Maxeke Johannesburg Academic Hospital and to evaluate the associated costs.

Methods: A retrospective study was conducted (June to December 2019) to identify the RR and rejection criteria of HIV serology samples throughout the total testing process. Descriptive analysis using percentages and frequencies was used to analyse the RR by phase, health establishment, ward and healthcare professional. A cost analysis incorporating minor and major costs was modelled in each phase of testing, and the total cost of rejections was calculated.

Results: A total of 6678 tests were received, and 738 were rejected $(R R=11.1 \%)$. The preanalytical phase contributed significantly to the overall $R R$, with the requirement of a separate sample $(57.44 \%)$ the most common reason for rejection. The total cost per rejected test was $\$ 2.47$, which amounted to a total rejection cost of $\$ 197.55$, of which $\$ 158.18$ was caused by the pre-analytical rejection criteria.

Conclusion: High RR of HIV tests were noted, resulting in significant cost wastage. Identification and analysis of rejections must be implemented across all laboratories to improve the efficiency of testing, provide a cost-saving benefit and maintain high laboratory standards.

Keywords: HIV; rejection rates; cost analysis; laboratory; diagnostics.

\section{Introduction}

HIV remains a leading cause of increased morbidity and mortality, especially in Southern Africa. Despite active measures to control the course of this disease, over 7 million individuals are living with HIV in South Africa. ${ }^{1}$ The Joint United Nations Programme on HIV / AIDS (UNAIDS) 95-95-95 goals highlight the role of diagnostic testing as one of the main strategies in controlling this pandemic. ${ }^{2}$ Providing accurate and reliable results timeously has proven to have positive outcomes in the management of HIV-infected individuals. ${ }^{3}$ In line with the 95-95-95 targets, South Africa adheres to the universal test and treat strategy. As such, the laboratory has a responsibility to provide quality results to promote patient safety. A constant increase in test demand results in increased workload, which leads to inefficiencies within the laboratory and healthcare facility. As a result, laboratory accountability for patient safety has been highlighted in recent studies and should include the monitoring and analysis of key quality indicators such as rejection rates (RR). ${ }^{4}$ This can be tailormade to accommodate different laboratories; however, when doing so, pre-existing limitations such as laboratory design, infrastructure, personnel and operating processes must be considered.

The rejection of a sample has detrimental consequences for the laboratory, health facility and individual tested. This can be reflected in delayed turnaround times, reduced efficiency, poor workflow, cost implications, missed or delayed diagnostic opportunities and loss to follow-up..$^{5,6}$ A rejected test caused by laboratory error or an HIV test that is incorrectly requested in a setting such as the early infant diagnosis (EID) programme can be a major pitfall in achieving targets that aim to reduce new paediatric infection rates (0-24 months). Several studies reported that a significant number of rejected tests are not repeated (once-off occurrence) and can account for up

Copyright: @ 2022. The Authors. Licensee: AOSIS. This work is licensed under the Creative Commons Attribution License. 
to a $12 \%$ increased chance of inappropriate patient care. ${ }^{7} \mathrm{~A}$ projected error rate of this magnitude in the South African context can apply tremendous pressure to the already financially constrained and resource-burdened public health sector, especially in key age categories such as the paediatric population. Recent literature reports several factors contributing to increased RR throughout the total testing process, including haemolysis, the mislabelling of samples and inappropriate sample collection. ${ }^{8}$ The total testing process consists of three phases - (1) pre-analytical, (2) analytical and (3) post-analytical - with the majority of rejections occurring in the pre-analytical phase. ${ }^{9}$

With the rise in the burden of diseases and the emergence of novel pathogens, there has been an increased need for diagnostic testing; however, this need has not been met with the necessary financial resources. To support the increase in testing, there is a need to review cost-saving strategies and how they will benefit the laboratory as well as the patient. A costing analysis that used the Markov probability model suggested a total loss of $\$ 357.15$ per hospital patient. ${ }^{10}$ Similarly, a number of studies have estimated pre-analytical RR costs to range between $\$ 160.00$ and $\$ 225.00$ per month. ${ }^{10,11}$ These substantial costs can have a significant impact on the total hospital budget.

The primary aim of this study was to assess the HIV serology RR and reasons in the Department of Virology, National Health Laboratory Service (NHLS), Charlotte Maxeke Johannesburg Academic Hospital (CMJAH). The secondary aim was to undertake a costing analysis to illustrate the financial implications of these rejections in a typical South African health laboratory.

\section{Materials and methods Study setting}

This retrospective study was conducted for the period 01 June 2019 to 31 December 2019 in the Department of Virology of the NHLS based at CMJAH (Johannesburg, South Africa). The department provides a dedicated 24-h diagnostic service to CMJAH, a tertiary-level hospital, surrounding primary healthcare (PHC) facilities and both regional and district level hospitals.

\section{Specimen registration}

For HIV serology test requests, samples were registered on the laboratory information system (LIS) following standard operating procedures to capture all demographic, clinical and test request details provided on the laboratory request form. The test results are automatically downloaded to the LIS, and all laboratory data are stored in the NHLS corporate data warehouse (CDW).

\section{Test rejections and rejection criteria}

All rejection codes and descriptions were defined by the local laboratory and the NHLS expert committees. Electronic gatekeeping of samples was included as a rejection criterion in this study, defined as any HIV serology sample requested before the minimum request interval time had elapsed. The pre-analytical phase included all processes from sample collection to receipt at the CMJAH NHLS receiving office. All processes that pertained to the performance of the test were assigned to the analytical phase, whereas the post-analytical phase involved the analysis, interpretation and authorisation of the test results.

\section{Lookup table}

For each rejection, a rejection code is entered into the LIS to indicate to the requesting healthcare practitioner the reason for not performing the test, for example 'specimen insufficient' (SPINS). Because of the vast number of rejection codes reported, a lookup table was developed using Microsoft Excel (Redmond, California, United States) to assign the rejection status (rejected/not rejected) and phase of testing (pre-analytical, analytical or post-analytical). Lookup tables were then used to group assigned codes of large data sets with similar information, avoiding manual coding (Table 1).

\section{Data analysis}

An NHLS-specific test code for HIV serology was used to extract data from the CDW for the 6 months (01 June to 31 December 2019). All rejected tests and the reasons for rejection were captured on the LIS and downloaded to the CDW database. The data extract also included the following variables: (1) age, (2) facility name, (3) referring healthcare professional, (4) rejection code, (5) rejection reason description, (6) date of collection, (7) date of rejection, (8) ward code and (9) ward description. Age was stratified into three categories - infants and toddlers (0-24 months), children and adolescents (2-18 years) and adults ( $>18$ years) - and the total number of rejections per age group was calculated. We used the facility name to assign the health establishment type (PHC facility or hospital). Similarly, we used ward descriptions to assign the following ward types: (1) medical, (2) trauma and casualty, (3) surgical, (4) intensive care unit (ICU), (5) paediatrics, (6) obstetrics and gynaecology and (7) antiretroviral (ARV) clinic. Experts read the ward description and assigned the ward type; for example 'Area 165 Medical Casualty CAS' was assigned as the trauma and casualty ward.

Requisitioner information, which included the healthcare practitioner's name and professional society registration details (South African Nursing Council [SANC] or Health Professions Council of South Africa [HPCSA]), was used to assign the following professional types: (1) nurse, (2) medical

TABLE 1: Example of some rejection codes and rejection reasons used to assign the rejection status and rejection phase values in a lookup table for HIV serology samples.

\begin{tabular}{llll}
\hline Rejection code & Rejection reason description & Rejection status & Rejection phase \\
\hline RSEP & Require separate specimen & Rejected & Pre-analytical \\
SPINS & Specimen insufficient & Rejected & Pre-analytical \\
NDLE & Not done: lab error & Rejected & Analytical \\
ONCOR & Not done: non-reportable result & Rejected & Post-analytical \\
CEGK & Electronic gatekeeping & Rejected & Pre-analytical \\
\hline
\end{tabular}


intern (IN) and (3) medical practitioner (MP). For example, requisitioner details that included SANC, IN and MP numbers were assigned as a nurse, intern and medical practitioner, respectively. Data that did not include the rejection reason description could not be categorised and were excluded from this study.

\section{Rejection rate calculations}

The RR were calculated using the formula:

$$
\left(\mathrm{RR}=\frac{\text { Rejections }}{\text { Total test volume }} \times 100\right)
$$

and reported as a percentage. The RR were analysed by process phase, health establishment, ward and healthcare professional.

\section{Cost analysis}

The cost analysis was performed to determine the cost per rejection for each phase of testing. All costs were obtained in South African rands (ZAR) and converted to United States dollars (USD) using an exchange rate of $14.60 / \$ 1.00$. The accounting stance was assumed to be the provider of diagnostic services, and costs associated with overheads and laboratory management were excluded. For the pre-analytical phase, data generated from a local study that conducted a top-down costing of historical expenditure data for the 2019-2020 financial period for the CMJAH receiving office were used (\$0.77 per registration). A pre-analytical cost per test was calculated using the assumption that on average 3.5 tests are requested per registration.

For the analytical phase (pre-analytical cost + analytical cost), the cost per test associated with the analyser, staffing, reagents and test consumables was determined. These costs were obtained from the Oracle Enterprise Resource Planning (ERP) system of the NHLS (Box 1). The Roche Cobas 8000 modular analyser (module e602; Roche Diagnostics, Basel, Switzerland) was provided through a service placement agreement, and thus the costs associated with the outright purchase, maintenance and servicing of the instrument are included in the reagent cost. All costing data were captured in Microsoft Excel for analysis.

For staffing costs (medical technologist - C2 grade), given the short sample preparation time, the time to perform the test (in minutes) was multiplied by the annual cost per minute, based on the assumption that HIV serology testing is offered

BOX 1: Itemised list of resources within the analytical phase of testing.

\begin{tabular}{l} 
Itemized list of resources \\
HIV combi reagent and calibrator \\
HIV control \\
Clean cell buffer $\mathrm{M} 2 \times 2 \mathrm{~L}$ \\
ProCell M \\
Probe clean M \\
Probe wash M \\
Assay tip/cup and waste box \\
Medical technologist (C2) $(5 \mathrm{~min})$ \\
\hline
\end{tabular}

on a 365-day running cycle (annual cost / 365). Similarly, for the post-analytical phase, the average time $(3 \mathrm{~min})$ for a registrar (D1 grade) to review and authorise the result on the LIS was used. All staff salaries were based on the NHLS cost to company (CTC) salary scales.

To calculate the total cost of rejection, the cost per test was multiplied by the total number of HIV serology rejections. Corporate warehouse data were provided as a CSV file used for preliminary analysis in Microsoft Excel. Descriptive analysis was conducted using TIBCO Statistica version 13.5.0 analytical software (California, United States).

\section{Ethical considerations}

The study was approved by the Human Research Ethics Committee, University of the Witwatersrand (clearance certificate number M201117).

\section{Results}

A total of 6678 HIV serology samples were received for the study period, of which 738 samples were rejected $(11.1 \%)$. Among the rejected group, a mean age of 32 years was reported (standard deviation: 20.57), with the majority attributed to the adult population $(560 / 738 ; 75.88 \%)$. Within

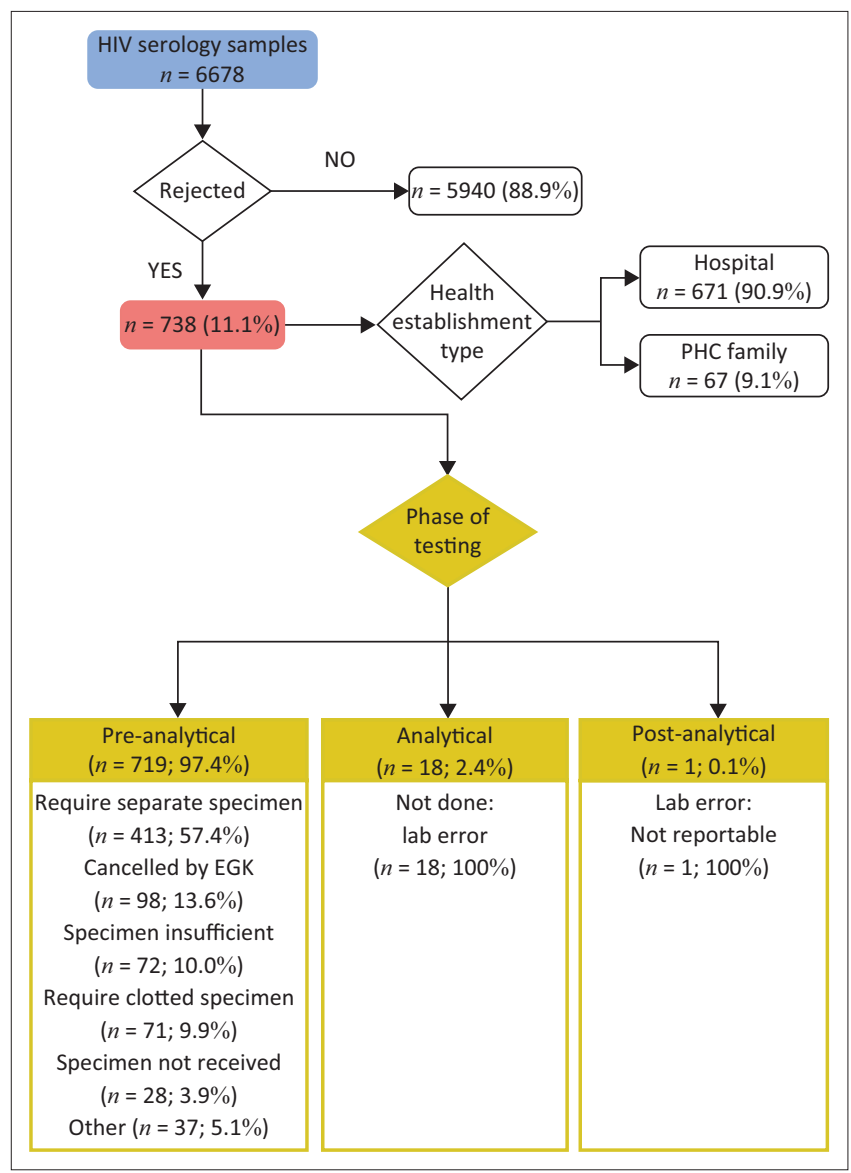

EGK, electronic gatekeeping; PHC, primary health care.

FIGURE 1: Rejection rates and criteria throughout the total testing process for HIV serology samples performed at the Department of Virology, National Health Laboratory Service, Charlotte Maxeke Johannesburg Academic Hospital, South Africa, between 01 June 2019 and 31 December 2019. 


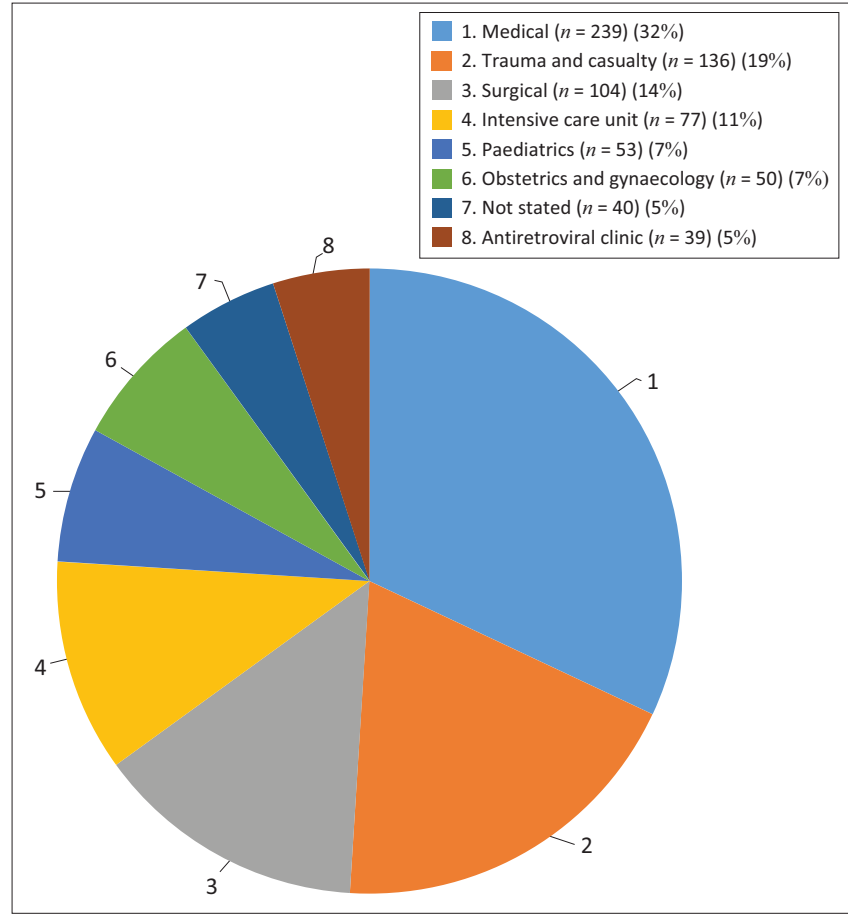

FIGURE 2: Pie chart displaying the percentage of HIV serology sample rejections by ward type at the Department of Virology, Charlotte Maxeke Johannesburs Academic Hospital, South Africa between 01 June 2019 and 31 December 2019. The intensive care unit metric includes both the adult and paediatric age categories.

the age group of $0-24$ months, there were 106 rejected samples (14.36\%). There were $671(90.92 \%)$ and $67(9.08 \%)$ rejected samples from hospitals and PHC facilities, respectively (Figure 1).

The majority $(719 / 738 ; 97.4 \%)$ of rejections occurred in the pre-analytical phase, where most samples $(413 / 719 ; 57.4 \%)$ were rejected because of the requirement for a separate sample (Figure 1). This was followed by rejections as a result of electronic gatekeeping (13.6\%), SPINS (10\%), requiring a clotted sample (9.9\%) and specimen not received (3.9\%). Other $(5.1 \%)$ reasons for sample rejection in the pre-analytical phase included mislabelling, incomplete healthcare worker or patient information, and unsuitable samples. Eighteen (2.43\%) samples were rejected in the analytical phase because of a number of laboratory errors, such as poor sample integrity, and one $(0.13 \%)$ in the post-analytical phase because of a non-reportable result.

Based on the ward type, the medical unit had the highest number of rejections $(239 / 738 ; 32 \%)$ followed by trauma and casualty $(136 / 738 ; 19 \%)$, surgical $(104 / 738 ; 14 \%)$ and ICU, which accounted for $11 \%(77 / 738)$ (Figure 2$)$. The requirement of a separate sample was the most common reason for rejection across all departments, health facilities and age groups. Within this criteria, hospital samples accounted for $375 / 413$ (90.80\%) and clinics for 38/413 (9.20\%) rejections. Transport of samples was a rejection criterion associated with clinic samples only $(3 / 67 ; 4.47 \%)$.

Further analysis by health establishment and healthcare profession type revealed that, at hospitals, the majority of
TABLE 2: Number of HIV serology samples rejected, by health establishment and healthcare professional type.

\begin{tabular}{llcc}
\hline $\begin{array}{l}\text { Health establishment } \\
\text { type }\end{array}$ & $\begin{array}{l}\text { Healthcare } \\
\text { professional type }\end{array}$ & \multicolumn{2}{c}{$\begin{array}{c}\text { Number of rejected } \\
\text { samples }(\boldsymbol{N}=\mathbf{7 3 8})\end{array}$} \\
\cline { 3 - 4 } & & $\boldsymbol{n}$ & $\mathbf{\%}$ \\
\hline Hospital & Medical practitioner & 543 & 80.92 \\
& Intern & 100 & 14.90 \\
& Nurse & 16 & 2.38 \\
& Clinical associate & 12 & 1.80 \\
Total & - & 671 & 100.00 \\
Primary health care & Nurse & 53 & 79.10 \\
facility & Medical practitioner & 11 & 16.41 \\
& Intern & 3 & 4.49 \\
Total & - & 67 & 100.00 \\
\hline
\end{tabular}

Note: Data are reported for the Virology Department at the Charlotte Maxeke Johannesburg Academic Hospital, South Africa, between 01 June 2019 and 31 December 2019.

$\dagger$, 'Medical practitioner' includes community service officer, medical officer, registrar and consultant.

TABLE 3: Itemised cost per sample for each phase of testing for HIV serology rejections.

\begin{tabular}{lc}
\hline Item in each phase of testing & Cost per sample (USD) \\
\hline Pre-analytical & $\mathbf{0 . 2 2}$ \\
Collection and registration & 0.05 \\
Laboratory equipment & 0.01 \\
Staff & 0.13 \\
Operating costs & 0.03 \\
Analytical & 1.83 \\
HIV reagent pack & 1.04 \\
Buffer & 0.26 \\
Waste consumables & 0.24 \\
Staff & 0.29 \\
Post-analytical & $\mathbf{0 . 4 2}$ \\
Staff & 0.42
\end{tabular}

USD, United States dollars.

TABLE 4: Determining the total cost of rejections for HIV serology testing across the three phases of testing.

\begin{tabular}{lcccc}
\hline Phase of testing & $\begin{array}{c}\text { Cost of } \\
\text { rejection per } \\
\text { sample (USD) }\end{array}$ & \multicolumn{2}{c}{ Number of rejections } & \multirow{2}{*}{$\begin{array}{c}\text { Total cost of } \\
\text { rejections } \\
\text { (USD) }\end{array}$} \\
\hline Pre-analytical & 0.22 & 719 & $\%$ & 158.18 \\
Analytical & 2.05 & 18 & 17.2 & 36.90 \\
Post analytical & 2.47 & 1 & 0.2 & 2.47 \\
\hline Total & $\mathbf{2 . 4 7 \dagger}$ & $\mathbf{7 3 8}$ & $\mathbf{1 0 0 . 0}$ & $\mathbf{1 9 7 . 5 5}$ \\
\hline
\end{tabular}

USD, United States dollars.

$\dagger$, The total cost is calculated incorporating the itemized cost in all three phases of the tota testing process (based on a single rejected sample).

rejections were requested by medical practitioners $(543 / 671$; $80.92 \%)$. In contrast, interns, nurses and clinical associates accounted for the remaining 19\%. For PHC facilities, $79.1 \%$ of rejections were requested or collected by nursing staff (Table 2).

Table 3 summarises the itemised costs for HIV serology by testing phase. The total cost per sample for the pre-analytical phase was calculated to be $\$ 0.22$. The bulk of the preanalytical costs was for staff, at $\$ 0.13$ per sample. For the analytical phase, a cost per test of $\$ 1.83$ was reported. The HIV reagent pack contributed $\$ 1.04$, compared to $\$ 0.50$ for buffer and waste consumables. The staff cost contributions for the analytical and post-analytical phases were \$0.29 and $\$ 0.42$, respectively (Table 3).

The total cost per sample was calculated to be $\$ 2.47$ across the three phases of testing. The total cost of rejections was $\$ 197.55$ (Table 4). The pre-analytical phase contributed $82.6 \%$ of the 
total cost of rejections (\$158.18), followed by the analytical phase, with $17.2 \%$ (\$36.90). Given the single post-analytical rejection, the total cost for that phase of testing was $\$ 2.47$.

\section{Discussion}

In this study, we assessed the rates, reasons and cost of rejections for HIV serology tests at the Department of Virology at an academic hospital in South Africa over 6 months in 2019. Overall RR of 3.6\% were reported for all test requests received during 2019 (CMJAH NHLS statistics, unpublished). This is consistent with other studies that reported similar average RR $(0.1 \%-3.49 \%){ }^{12,13}$ However, the HIV serology RR of $11.1 \%$ reported in this study are significantly higher than reported rates and not aligned with the accepted internal test RR of $<5 \%$ set for Virology.

In the laboratory setting described, multiple reasons for rejection throughout the total testing process were noted. As reported in other studies, the pre-analytical phase was identified as the main phase of rejection, where 1 in 10 patients had a missed or delayed diagnosis, predominantly as a result of the requirement for a separate sample. This criterion is consistently noted as the most common reason for rejection in the data described here and highlights a gap in training on HIV serology sample collection for healthcare practitioners.

In addition, despite current EID guidelines, which recommend that an HIV DNA polymerase chain reaction (PCR) test be done from birth to 18 months of age, $14 \%$ of rejected tests fell within this age group. Our data identified that HIV serology tests are incorrectly requested within this age group, indicating a need for clinical training on guidelines and laboratory requirements for testing. It further underlines the importance of routine monitoring and analysis of the rejection criteria as a key quality indicator for continuous improvement.

This study also illustrates the laboratory's acceptability criteria and how strictly they are adhered to. Within the receiving laboratory, all staff are guided by the standard operating procedures for registration of a sample and the criteria for rejection. A large number of rejection criteria also fall within the clinical domain, over which the laboratory may not necessarily have influence and which requires tighter control with regard to the collection and requisition of samples by clinical departments. Other studies have shown similar findings, in that a large number of errors (preanalytical) occur outside the laboratory and are caused by actions predominantly by the healthcare workers. ${ }^{14}$ This is attributable to frequent staff rotation and substandard training. It is clear from the data of this study which healthcare personnel requested or drew samples that led to rejections and would benefit from additional training. All these aspects are clearly described in the NHLS handbook provided to hospitals and clinics. As such, these errors could have easily been avoided, subsequently eliminating a large percentage of rejections. It is important to note that the rejected tests originate mainly from the medical, trauma and casualty, and surgical wards, where timeous and accurate HIV results are important for clinical management of patients because of the high burden of HIV in South Africa.
In response to these findings, despite the lack of standardised rejection criteria amongst laboratory networks, appropriate corrective and preventative actions can be implemented, monitored and assessed. These include regular staff training and routine competency assessments directed towards key receiving office staff and healthcare workers. These training mechanisms will provide the greatest outcome in terms of RR reduction but should also be extended to all healthcare and laboratory workers. Laboratory manuals targeting key issues such as requesting a separate sample for HIV testing can avoid delays in turnaround time and patient management. Training on the current HIV and EID guidelines, as well as providing itemised rejections for a specific test set by ward, may also aid in the regular monitoring and evaluation of procedures, which will ensure good diagnostic practices.

Reduced RR may lead to improved patient care. ${ }^{15}$ The impact of rejecting samples also has financial implications. The current study findings assessed the cost implications of these rejections. For one laboratory, an annual cost of $\$ 383.03$ was reported. ${ }^{16}$ When these data are extrapolated for national HIV serology testing across the NHLS for the 2019-2020 financial period (assuming 11.1\% RR), the total cost of rejections amounts to $\$ 122$ 295. This signifies a substantial cost burden to the health system that could be avoided. Furthermore, the cost of rejections must be seen as a contributing loss within the broader healthcare system. Improving the efficiency of testing will have a twofold benefit, firstly providing a significant contribution to healthcare resource savings and secondly improving a key quality indicator that plays a pivotal role in maintaining high laboratory standards. ${ }^{17,18}$ As the rejections occurred primarily within the pre-analytical phase in this study, it affirms that measures put in place through detailed rejection criteria by the NHLS also served to reduce the costs associated with performing unnecessary test or tests with compromised quality through the use of shared samples. ${ }^{19}$

The study limitations included a small number of unspecified rejection reasons from the data extract, as well as the rejection reasons being grouped into similar categories. This may have resulted in non-specific rejection codes being used that therefore did not necessarily accurately describe the rejection. The data from this study only take into consideration HIV laboratorybased testing and exclude a large volume of point-of-care testing performed at other health establishments. However, because of the availability of such data, this study recognises an opportunity to improve the analysis of RR if the data are managed properly and categorised in a more user-friendly manner. This will allow for laboratories to monitor their data over shorter time intervals and will lead to the timely identification of problem areas. This will encourage proactive measures to be implemented in order to reduce the overall number of rejections.

\section{Conclusion}

There are substantial data to suggest that inappropriate test use, rejections and repeat testing contribute to increased laboratory expenditure and unfavourable patient outcomes. Identification of the key $\mathrm{RR}$ is an additional tool to monitor laboratory 
efficiency, improve service delivery and identify areas in the testing process that need intervention through corrective actions. Limiting rejections in the laboratory will save significant costs and time and improve the clinical utility of diagnostic tests, which will benefit the laboratory, patients and the healthcare system.

\section{Acknowledgements}

The authors acknowledge and thank the Department of Virology and staff who assisted in the processing of samples. The NHLS is acknowledged for granting access to the data used in this study.

\section{Competing interests}

The authors acknowledge no financial or personal relationships that may have inappropriately influenced them during the write-up of this article.

\section{Authors' contributions}

B.R. and Z.M. conceptualised the study. B.R. executed the research and data analysis. The first draft was reviewed by Z.M. N.C. provided input into the cost model and addition of key resources. Z.M, N.C and F.T reviewed the subsequent drafts, provided critical revision and feedback, and contributed to the overall standard of work.

\section{Funding information}

The authors received no financial support for the research, authorship, and/or publication of this article.

\section{Data availability}

The raw data were generated by the National Health Laboratory Services Corporate Data Warehouse. Derived data supporting the findings of this study are available from the corresponding author, B.R., upon suitable and fair request.

\section{Disclaimer}

The views expressed in this article belong to the authors and do not represent an official position of the associated institutions.

\section{References}

1. Maharaj P, Baijnath S, Naidoo P. Knowledge and practices of HIV infected patients regarding medicine disposal among patients attending public ARV clinics in KwaZulu Natal, South Africa. BMC Public Health. 2020;20(1):884. https://doi. org/10.1186/s12889-020-09018-4

2. Bain LE, Nkoke C, Noubiap JJN. UNAIDS 90-90-90 targets to end the AIDS epidemic by 2020 are not realistic: Comment on 'Can the UNAIDS 90-90-90 target be achieved? A systematic analysis of national HIV treatment cascades'. BMJ. 2017;2(2):e000227. https://doi.org/10.1136/bmjgh-2016-000227

3. Lippi G, Banfi G, Buttarello M, et al. Recommendations for detection and management of unsuitable samples in clinical laboratories. Clin Chem Lab Med. 2007;45(6):728-736. https://doi.org/10.1515/CCLM.2007.174

4. Baker A. Specimen rejection in laboratory medicine: Necessary for patient safety? Biochem Med. 2011;25(3):130-144. http://doi.org/10.11613\%2Fbm.2015.037

5. Gupta V, Negi G, Harsh M, Chandra H, Agarwal A, Shrivastava V. Utility of sample rejection rate as a quality indicator in developing countries. J Natl Accredit Board Hosp Healthc Provider. 2015;2:30. https://doi.org/10.4103/2319-1880.160245

6. Lippi G, Blanckaert N, Bonini P, et al. Haemolysis: An overview of the leading cause of unsuitable specimens in clinical laboratories. Clin Chem Lab Med. 2008;46(6):764-772. https://doi.org/10.1515/CCLM.2008.170

7. Jacobsz LA, Zemlin AE, Roos MJ, Erasmus RT. Chemistry and haematology sample rejection and clinical impact in a tertiary laboratory in Cape Town. Clin Chem Lab Med. 2011;49(12):2047-2050. https://doi.org/10.1515/CCLM.2011.743

8. Plebani M. Exploring the iceberg of errors in laboratory medicine. Clin Chim Acta. 2009;404(1):16-23. https://doi.org/10.1016/j.cca.2009.03.022

9. Lippi G, Guidi GC. Risk management in the preanalytical phase of laboratory testing. Clin Chem Lab Med. 2007;45(6):720-727. https://doi.org/10.1515/ CCLM.2007.167

10. Green SF. The cost of poor blood specimen quality and errors in preanalytical processes. Clin Biochem. 2013;46(13-14):1175-1179. https://doi.org/10.1016/j. clinbiochem.2013.06.001

11. Mehdi SR, Ahmad S, Zaidi N. Assessment of laboratory errors and best laboratory practices. Int J Bioassays. 2016;5(7):4704. https://doi.org/10.21746/ijbio.2016.07.008

12. Kamal F, Wan Mohd Saman WA, Adbul Monir M. Analysis of sample rejection and the impact on quality of care in patients in a single tertiary healthcare facility in Malaysia. Environ Proc J. 2020;5(13):175-181. https://doi.org/10.21834/e-bpj. v5i13.2104

13. Lippi G, Chance JJ, Church S, et al. Preanalytical quality improvement: From dream to reality. Clin Chem Lab Med. 2011;49(7):1113-11126. https://doi.org/10.1515/ CCLM.2011.600

14. Gunnur Dikmen Z, Pinar A, Akbiyik F. Specimen rejection in laboratory medicine Necessary for patient safety? Biochem Med. 2015;25(3):377-385. https://doi org/10.11613/BM.2015.037

15. Lippi G, Plebani M, Graber ML. Building a bridge to safe diagnosis in health care: The role of the clinical laboratory. Clin Chem Lab Med. 2016;54(1):1-3. https:// doi.org/10.1515/cclm-2015-1135

16. Rooper L, Carter J, Hargrove J, Hoffmann S, Riedel S. Targeting rejection: Analysis of specimen acceptability and rejection, and framework for identifying interventions in a single tertiary healthcare facility. J Clin Lab Anal. 2017;31(3): 1-8. https://doi.org/10.1002/jcla.22060

17. Hammerling JA. A review of medical errors in laboratory diagnostics and where we are today. Lab Med. 2012;43(2):41-44. https://doi.org/10.1309/LM6ER9WJR1IHQAUY

18. Lippi G, Blanckaert N, Bonini P, et al. Causes, consequences, detection, and prevention of identification errors in laboratory diagnostics. Clin Chem Lab Med. 2009;47(2):143-153. https://doi.org/10.1515/CCLM.2009.045

19. Lippi G, Plebani M, Simundic A-M. Quality in laboratory diagnostics: From theory to practice. Biochem Medica. 2010;20(2):147-153. https://doi.org/10.11613/ BM.2010.014 